\title{
A new in situ method to analyze mineral particle reactions in soils
}

SUPPORTING INFORMATION

\author{
Andreas Birkefeld, Rainer Schulin and Bernd Nowack \\ Institute of Terrestrial Ecology (ITÖ), Swiss Federal Institute of Technology Zurich (ETH), \\ Grabenstrasse 3, CH-8952 Schlieren, Switzerland
}

Contact: Bernd Nowack, Institute of Terrestrial Ecology (ITÖ), Swiss Federal Institute of Technology Zurich (ETH), Grabenstrasse 3, CH-8952 Schlieren, Switzerland Fax: ++41 1 6331123; Phone: +41 1 6336061; email: nowack@env.ethz.ch 


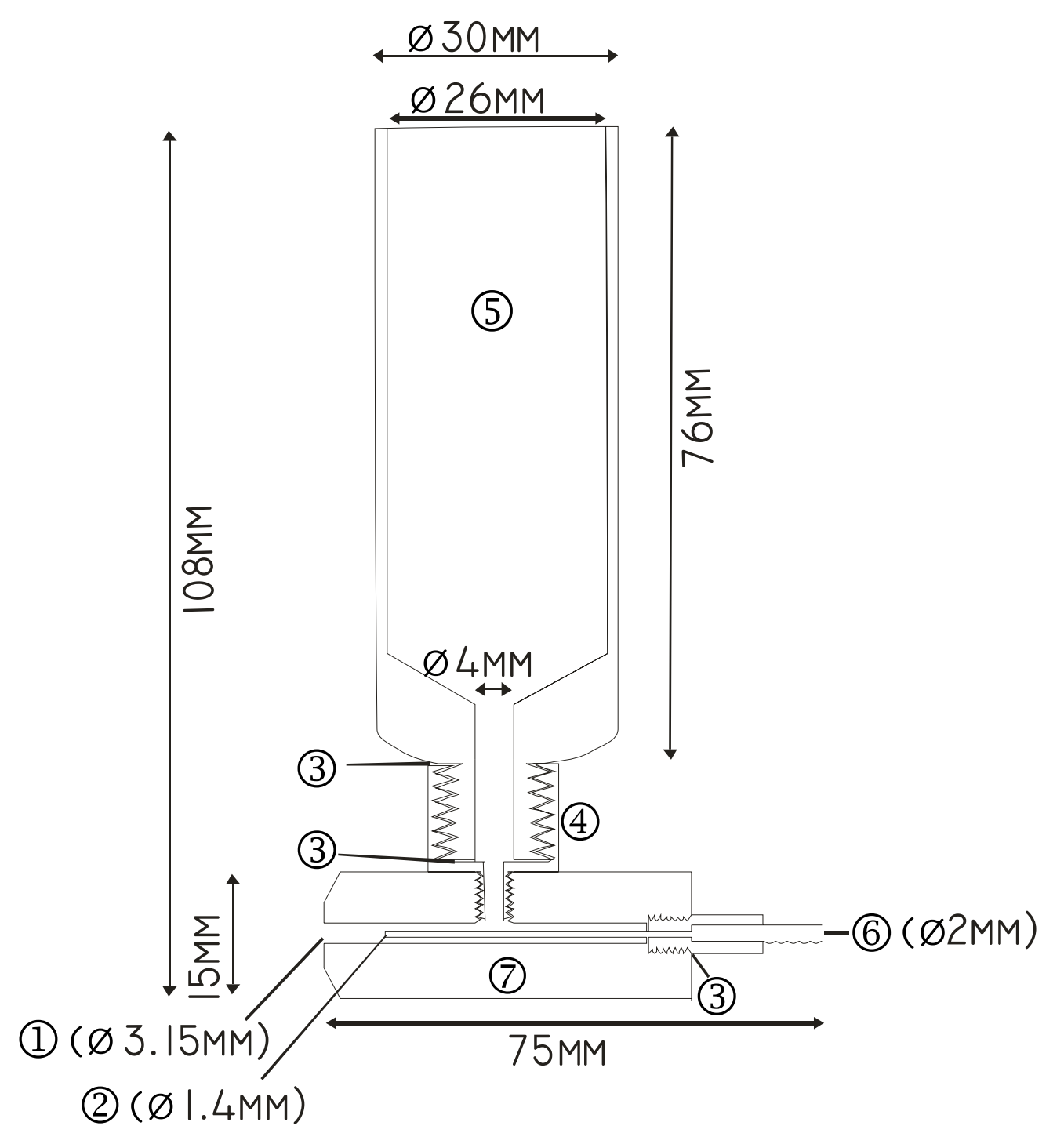

Figure 1S. Dust spray gun for particle application. (1) Exit orifice, (2) Compressed air nozzle, (3) Sealing gasket, (4) Reservoir holder (brass), (5) Particle reservoir (PMMA), (6) Compressed air inlet (brass), (7) Dust gun body (brass) 


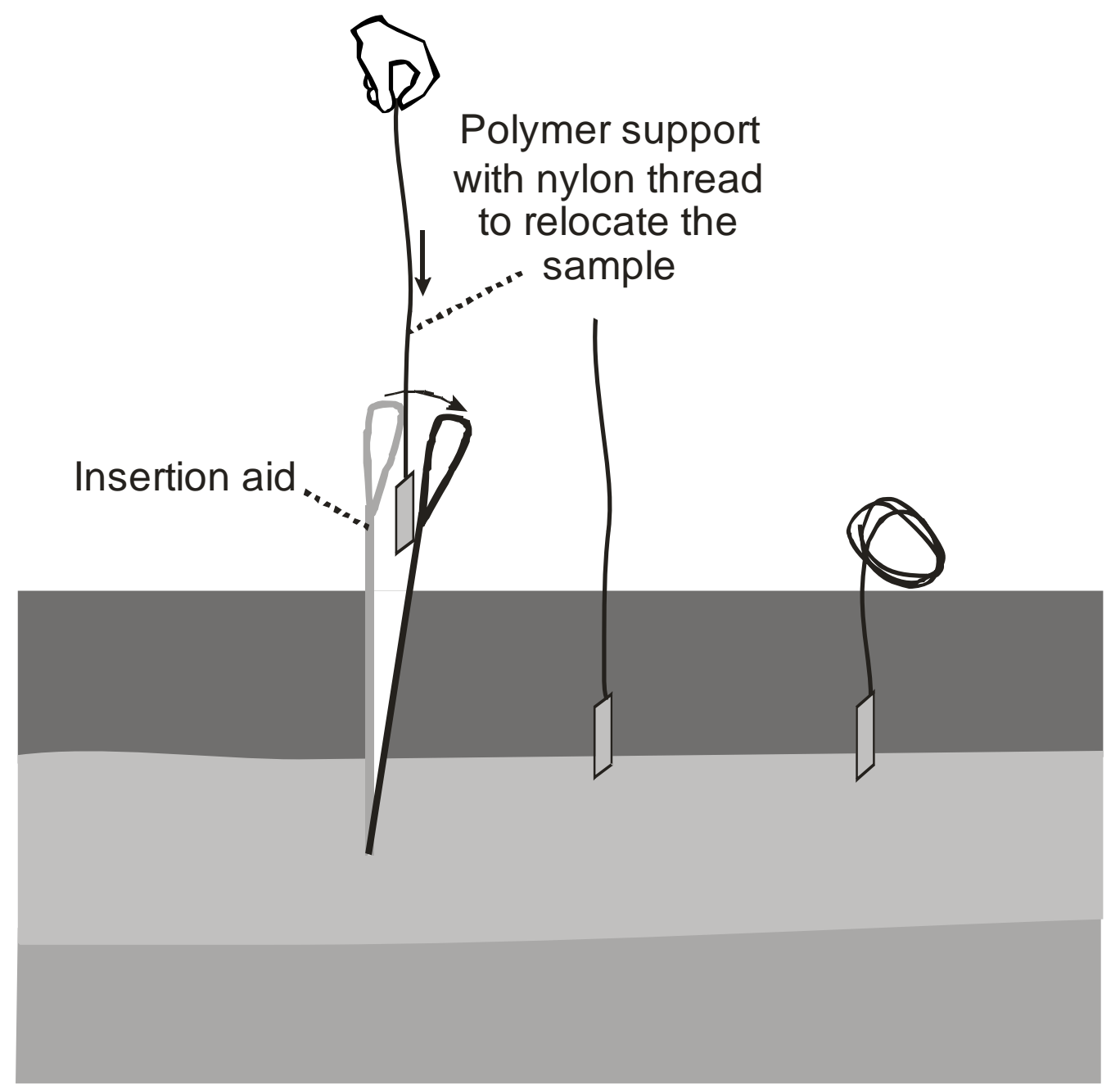

Figure 2S: Scheme of the sample insertion process. The insertion lancet is stuck into the soil and bend forward to open a small slit. The sample is inserted into the soil and the slit closed. The attached nylon thread helps to relocate the sample after the experiment. 


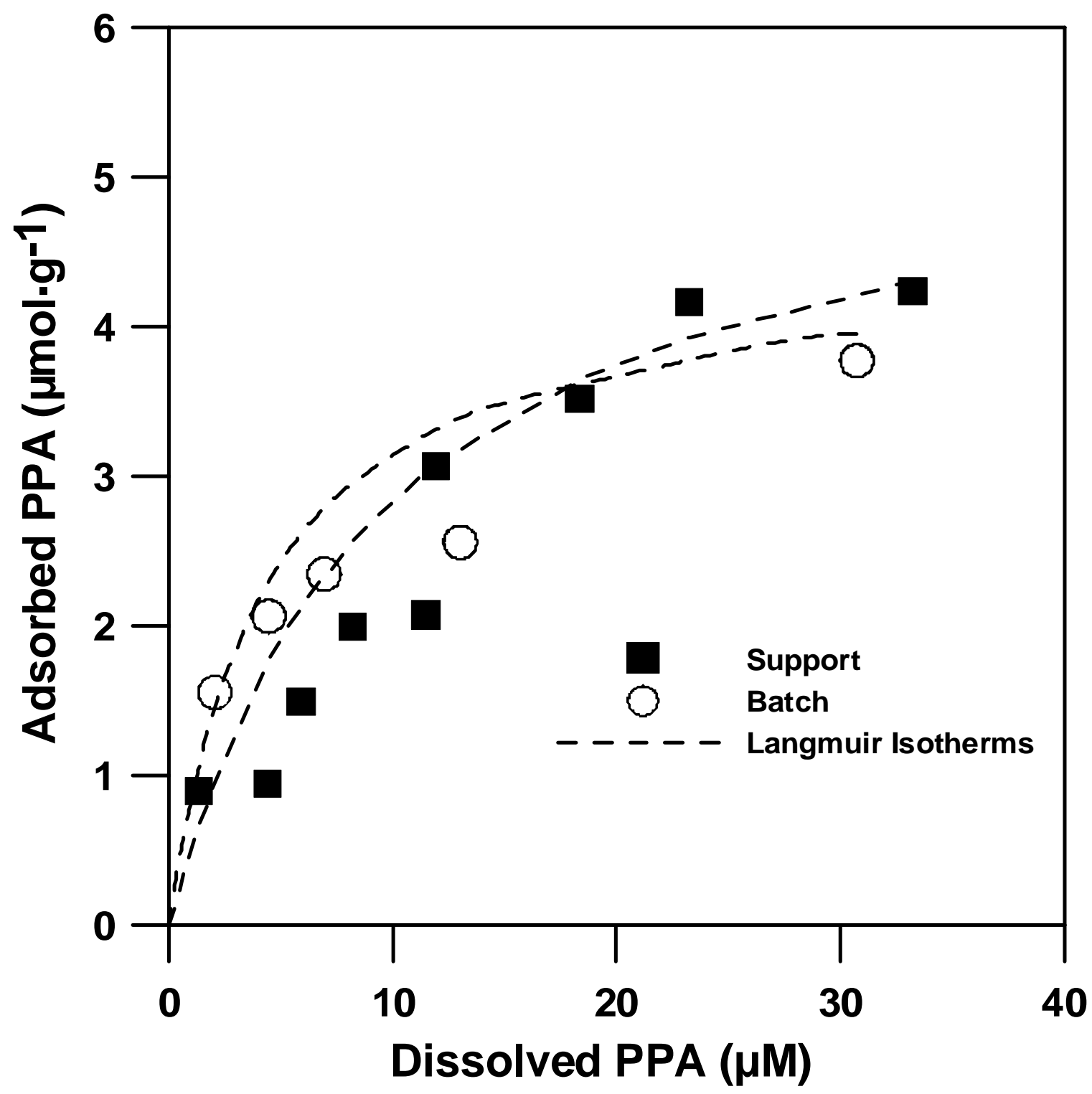

Figure 3S. Adsorption isotherms of phenylphosphonic acid (PPA), buffered at pH 7.1 with MES) on polymer supports covered with copper oxide (filled squares) and on copper oxide in bulk suspension (empty circles). The dashed lines are the calculated Langmuir isotherms 


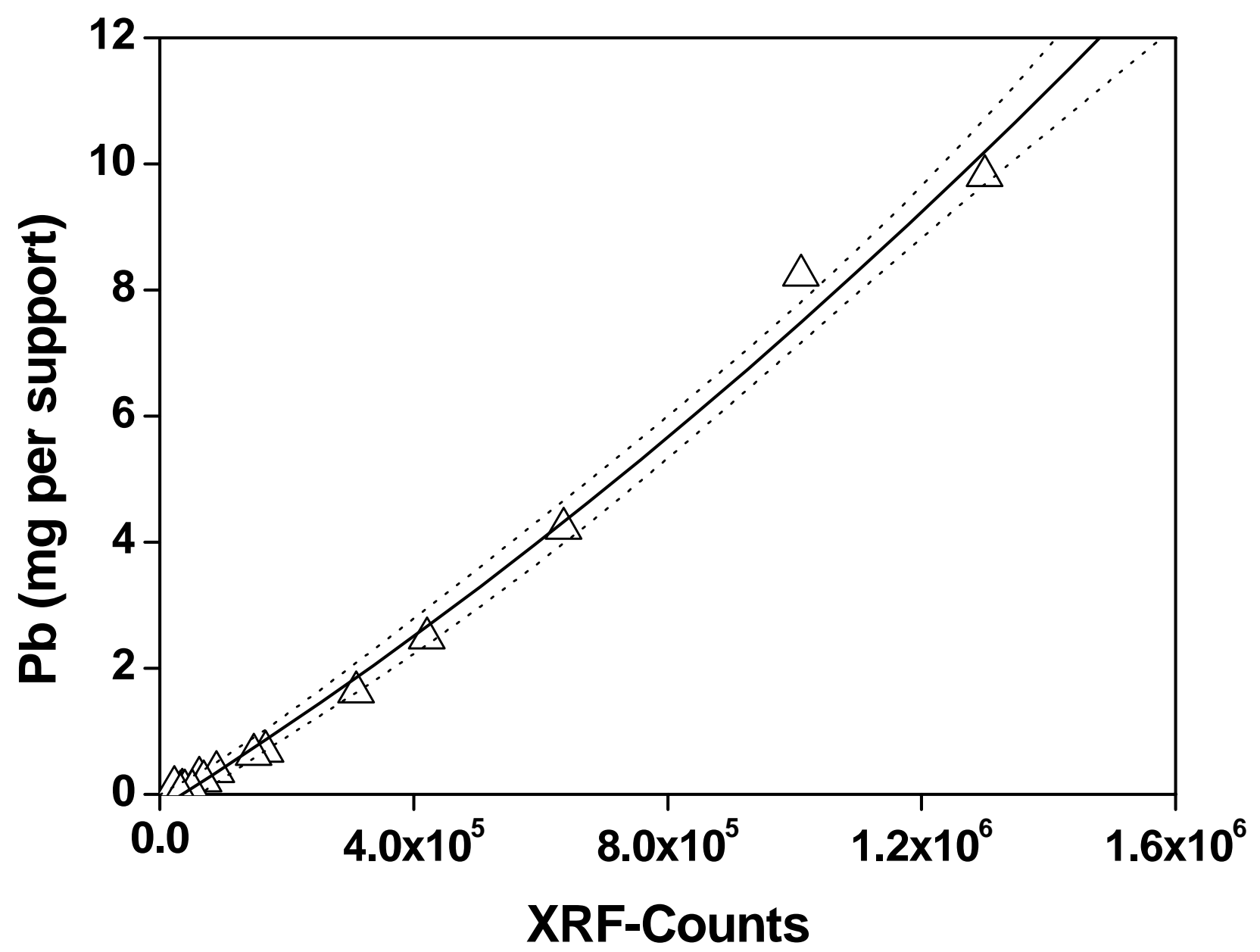

Figure 4S. Calibration of the XRF device for PbO-covered supports. The non-invasive XRF results (counts) can be converted directly into actual elemental concentrations per support (mg Pb·support ${ }^{-1}$ ) which was acquired by complete dissolution and AAS analysis. The different concentrations were achieved by partial dissolution of the particles. The lines represent the second order polynomial fit and the 95\% confidence interval. 